\title{
Glucocorticoid-induced delayed fracture healin
and impaired bone biomechanical properties in mice
}

This article was published in the following Dove Press journal:

Clinical Interventions in Aging

Yan-zhi Liu, ${ }^{1,2}$ Mohammed P Akhter, ${ }^{3}$ Xiang Gao, ${ }^{4}$ Xiaoyan Wang, ${ }^{2}$ Xiao-bei Wang, ${ }^{2}$ Gang Zhao, ${ }^{2}$ Xin Wei, ${ }^{2} \mathrm{Hao}-$ jun Wu, ${ }^{4}$ Hang Chen, ${ }^{4}$ Dong Wang, ${ }^{2}$ Liao Cui'

'Guangdong Key Laboratory for Research and Development of Natural Drugs, Guangdong Medical University, Zhanjiang, People's Republic of China; ${ }^{2}$ Department of Pharmaceutical Sciences, College of Pharmacy, University of Nebraska Medical Center, Omaha, NE, USA; ${ }^{3}$ Osteoporosis Research Center, Department of Medicine, Creighton University, Omaha, NE, USA; ${ }^{4}$ Stem Cell Research and Cellular Therapy Center, Department of Orthopaedics, Affiliated Hospital of Guangdong Medical University, Zhanjiang, People's Republic of China
Correspondence: Liao Cui Guangdong Key Laboratory for Research and Development of Natural Drugs, Guangdong Medical University, Room I I04, Wen-ming Dong Road

2\#, Zhanjiang, Guangdong 524023,

People's Republic of China

$\mathrm{Tel} / \mathrm{fax}+867592388305$

Email cuiliao@।63.com

Dong Wang

Department of Pharmaceutical Sciences, College of Pharmacy, University of

Nebraska Medical Center, PDD 3020,

Omaha, NE 68198-6125, USA

$\mathrm{Tel}+\mathrm{I} 4025591995$

$\mathrm{Fax}+\mathrm{I} 4025599543$

Email dwang@unmc.edu
Background: The objective of the study was to investigate the effects of glucocorticoid (GC) on the fracture healing process in a closed femur fracture mice model.

Materials and methods: Forty 12 -week-old female CD-1 mice were randomly allocated into four groups: healthy control and mice with prednisone exposure (oral gavage), $6 \mathrm{mg} / \mathrm{kg}$ / day (GC-L), $9 \mathrm{mg} / \mathrm{kg} / \mathrm{day}(\mathrm{GC}-\mathrm{M})$ and $12 \mathrm{mg} / \mathrm{kg} /$ day $(\mathrm{GC}-\mathrm{H})$. Three weeks after the initiation of prednisone dosing, closed femur fractures were created on prednisone-exposed mice and the healthy control. Prednisone administration was continued for 9 weeks post-fracture, and X-ray imaging was performed weekly to monitor the fracture healing process until the mice were euthanized. Necropsy was performed after 9 weeks and the fractured femurs were isolated and processed at necropsy for micro-CT and biomechanical property analysis. Another 20 mice (control and GC-H, 10 mice/group) were used for histology and micro-CT analysis at early time point (2-week post fracture) with continued prednisone exposure.

Results: The results showed that oral administration of prednisone for 3 months in this strain of mice could inhibit endochondral ossification and delay the healing process, especially hard callus formation (woven bone) and bone remodeling during healing. It also could significantly decrease bone biomechanical properties.

Conclusion: Long-term GC administration leads to significantly delayed fracture healing and impaired bone biomechanical properties. This mouse model may be used to systematically study the cellular and molecular mechanisms underlying fracture healing with GC treatment background and may also be used to study the influence of different therapeutic interventions for bone fracture healing.

Keywords: delayed healing, non-union, fracture, glucocorticoid, mouse, stiffness, toughness

\section{Introduction}

Approximately 18 million bone fractures occur in the USA each year. ${ }^{1}$ Although most heal normally, $\sim 10 \%$ of fractures are complicated by delayed healing or non-union, which often result in chronic pain, additional surgical procedures, impaired quality of life, reduced work productivity and higher healthcare costs. ${ }^{2}$ Delayed healing and nonunion are two major complications associated with fracture. Although they have some common characteristics, important clinical and genetic differences between the two exist and their molecular etiology largely remains unclear. The clinical appearance of fracture healing impairment is heterogeneous, and the contributing factors may include biomechanical destabilization, trauma, infection, hormone status, aging, concurrent diseases (eg, diabetes mellitus and rheumatoid arthritis), medication (eg, non-steroidal anti-inflammatory drugs and glucocorticoids [GCs]), smoking and alcohol abuse. ${ }^{3}$ 
GCs are extensively utilized in treating autoimmune diseases (eg, rheumatoid arthritis, systemic lupus erythematosus, allergies and asthma, etc.) and numerous other conditions (eg, adrenal insufficiency, heart failure, cancer and organ transplantation, etc.). Around $0.85 \%-0.9 \%$ of the general population aged $>18$ years in the UK and $1.2 \%$ of the US population aged $>20$ years have received systemic GCs. ${ }^{4}$ Long-term oral GCs prescriptions have increased by $34 \%$ over the past 20 years, ${ }^{5,6}$ with the highest age-stratified rate of $2.5 \%$ among people aged 70-79 years and the highest sex/agestratified rate of $3.05 \%$ among women aged $80-89$ years. ${ }^{7}$ The mean duration of oral GC use was 1,605.7 days, and $28.8 \%$ of oral GC users reported use for $>5$ years. ${ }^{4}$ Most patients with long-term GC exposure are at high risk of developing adverse events. One of major adverse events associated with GCs is significant deterioration of the skeleton (eg, osteoporosis and osteonecrosis). GCs may also affect the healing of fracture. The impact of GCs on the fracture healing process has been previously investigated. While the inhibitory effect of GCs on fracture healing seems logical, the outcomes from different animal studies but not all animal studies are consistent. A number of studies have demonstrated detrimental effects of GCs on fracture healing (eg, decreased callus volume, mineral content, the rate of fibroblasts and collagen accumulation and delayed endochondral ossification); ${ }^{8-11}$ other reports suggested that short-term GCs do not affect bone healing. The impact of GCs on fracture healing process may be dose and duration dependent. ${ }^{12-14}$ A single-dose and short-term regimen of GCs after fracture may be well tolerated; however, sustained dose and prolonged treatment could be deleterious to fracture healing. The effects of long-term GC exposure on fracture healing have not been fully investigated. To date, most of researches used rats to investigate the effects of GCs on fracture healing. ${ }^{15}$ A few mouse models of GC treatment-associated impaired fracture healing have been reported. Compared to larger animals, mice have the advantages of easy handling, low husbandry costs, short reproductive cycle and ease in genetic manipulation. In this study, we investigated long-term impacts of GC exposure on fracture healing in mice and characterized it as a GC-induced delayed fracture healing mouse model. This mouse model may be a valuable tool to investigate the pathophysiological mechanisms of GC-associated fracture healing impairment, and may also be used for screening and validation of therapeutic intervention for the pathology.

\section{Materials and methods}

\section{Animal care and study design}

Forty 12-week-old female CD-1 mice were purchased from Charles River Laboratories, Inc. (Kingston, NY, USA) and were divided into four groups (10 mice/group): healthy control and mice with prednisone (Acros Organics, Morris Plains, NJ, USA) exposure (oral gavage), $6 \mathrm{mg} / \mathrm{kg} / \mathrm{day}$ (GC-L), 9 mg/kg/day (GC-M) and $12 \mathrm{mg} / \mathrm{kg} /$ day (GC-H). As a frequently used GC in the clinical setting, daily oral prednisone was used to condition the animals 3 weeks prior to fracture surgery and was administered at 9 weeks postfracture. X-ray imaging was performed weekly to monitor the fracture healing process until euthanasia. The radiographs of the fractured callus area were quantified and analyzed weekly using ImageJ software (NIH, Bethesda, MD, USA). At euthanasia, the fractured femurs were isolated and processed for micro-CT and biomechanical property analysis (based on the results and analysis of X-ray image, only the mice from the control and $\mathrm{GC}-\mathrm{H}$ groups were used for the micro-CT and biomechanical property analysis).

To evaluate the effect of prednisone exposure on the callus microstructure and histological changes, 20 mice were obtained and randomized into two groups (10 mice/ group): healthy control and mice with prednisone exposure (12 mg/kg/d, GC-H). A closed femoral fracture was established as previously described. Different from the last experiment, the animals were exposed to prednisone only for 2 weeks post-fracture. The mice were then euthanized with the fractured femurs isolated and processed for micro-CT and histological analysis.

\section{Ethics statement}

All animal procedures described in this manuscript were approved by the Institutional Animal Care and Use Committee (IACUC) of the University of Nebraska Medical Center and the Animal Research Ethics Committee of Guangdong Medical University. All procedures were performed in accordance with Principles of Laboratory Animal Care (National Institutes of Health publication 85-23, revised in 1985).

\section{Surgical procedures for the establishment of closed fracture}

Three weeks after the initiation of prednisone dosing, closed femur fractures were created on prednisone exposed mice and the healthy control based on a previous report. ${ }^{16}$ Briefly, the mice were anesthetized with isoflurane $1 \%-1.5 \%$ inhalation with a $1.5 \mathrm{~L}$ oxygen flow rate. The region around the knee of the right leg was shaved with an electric shaver and scrubbed with Betadine ${ }^{\circledR}$ to prepare for surgery. A $3 \mathrm{~mm}$ medial parapatellar incision was made. The patella was dislocated to expose the femoral condyles. A 25-gauge needle was used to drill a hole into the femoral intramedullary canal at the intercondylar notch. A Faxitron MX-20 high-resolution X-ray 
system (Faxitron Bioptics, Tucson, AZ, USA) was used to confirm that the needle was inserted right into the femoral intramedullary canal. After X-ray imaging confirmation, the needle was cut and pushed deep into the marrow cavity of the femur. The needle served as internal fixation to stabilize the impending fracture. Subsequently the patella was repositioned, and the incision was closed using 4-0 synthetic absorbable suture for the muscle and non-absorbable suture for the skin layer. The skin suture was removed 10-14 days after the surgery. A closed diaphyseal fracture on the midshaft of the right femur was created using a modified method as described by Bonnarens and Einhorn. ${ }^{17}$ Specifically, by using a 3-point bending device with a $5 \mathrm{~mm}$ gap and a drop weight of $\sim 300 \mathrm{~g}$ from $15 \mathrm{~cm}$ height, or a similar manual setting was used to create the fracture. Therefore, a highly reproducible transverse fracture was created with minimal comminution and minimal angulation of the intramedullary pin. The animals were radiographed immediately postfracture induction to verify the creation of a mid-diaphyseal fracture. The $\mathrm{X}$-ray image system was equipped with an anesthesia system using isoflurane and the imaging procedures were performed within a maximum of $2 \mathrm{~min}$. After recovery from anesthesia, the animals were allowed unrestricted weight bearing. Twice-daily antibiotic (cefazolin sodium, $25 \mathrm{mg} / \mathrm{kg}$, s.c.) and analgesia (buprenorphine, $0.1 \mathrm{mg} / \mathrm{kg}$, s.c.) were given for 3 days following surgery according to the approved IACUC protocol.

\section{Micro-CT evaluation of fracture healing}

The collected fractured femurs were fixed in $10 \%$ formalin and processed for micro-CT analysis (SkyScan1172; Bruker, Kontich, Belgium). Each femur was scanned and 3D reconstructed with a voxel size of $5.5 \mu \mathrm{m}$. The X-ray tube voltage was $70 \mathrm{kV}$ and the current was set at $141 \mu \mathrm{A}$, with a $0.5 \mathrm{~mm}$ thick aluminum filter. Exposure time was $620 \mathrm{~ms}$, the X-ray projections were obtained at $0.4^{\circ}$ intervals with a scanning angular rotation of $180^{\circ}$ and 6 frames were averaged for each rotation. Three-dimensional reconstructions were performed using NRecon software. The position of each bone was corrected with DataViewer software. The region spanning of $5 \mathrm{~mm}$ (462 slices) on either side of the fracture line was selected as the region of interest (ROI). For the samples at the endpoint of 2 weeks post fracture, the ROI was carefully resized to a constant cube $\left(5 \mathrm{~mm}^{3}\right)$. For the samples at 9 weeks post-fracture, the callus size was measured by semiautomatic contouring with CTAn analysis software within the ROI. To standardize the analysis, we ensured to keep the constant ROI in the center for each sample, with the fracture line and fixation needle serving as horizontal and vertical points of reference, respectively. The following parameters were measured and calculated: callus bone volume to tissue volume (BV/TV), callus bone surface to volume (BS/TV) and connectivity density (Conn.D). Bone mineral content (BMC) and bone mineral density (BMD) were also analyzed with a calibration of 0.25 and $0.75 \mathrm{mg} / \mathrm{mm}^{3}$ standard hydroxyapatite blocks.

\section{Histological analysis of fracture healing}

After micro-CT analysis, the fracture samples (at 2 weeks post-fracture) were decalcified using $15 \%$ EDTA at $25^{\circ} \mathrm{C}$ for 30 days. The decalcification solution was changed daily. The specimens were subsequently paraffin embedded, sectioned $(5 \mu \mathrm{m})$ and stained with Safranin $\mathrm{O}$ and hematoxylin and eosin for histological evaluation. The ROI in the histological analysis was $3 \mathrm{~mm}$ below and above the fracture line. The total callus area (TCA), including chondrocyte and bone areas in the callus, was quantified with Image-Pro Plus 6.0 software (Media Cybernetics, Silver Spring, MD, USA) based on the histological staining. The ratio of bone/chondrocyte area in the TCA is also calculated.

\section{Evaluation of biomechanical properties of fracture healing}

The fractured femurs at 9 weeks post-fracture were isolated with fixation pins gently removed. The samples were frozen in saline in a freezer at $-20^{\circ} \mathrm{C}$. After being thawed to room temperature, the mid-shafts of the femurs were scanned by micro-CT with the same condition as already described. Midshaft cross-sectional size properties (area, second moment of area) were analyzed in the region spanning $1 \mathrm{~mm}$ on the mid-shafts of the femur. Subsequently, the specimens were tested for mechanical properties using an Instron 5543 Table Top System (Instron Limited, Canton, MA, USA). Each femur was placed on two lower supports that were $5 \mathrm{~mm}$ apart. Force was applied at the mid-diaphysis on the anterior surface so that the anterior surface was in compression and the posterior surface in tension. A load-displacement diagram for each test was created to determine structure strength properties (ultimate load, yield load and stiffness). The mid-shaft cross-sectional size obtained from the micro-CT analysis was used to calculate the normalized apparent material strength properties (ultimate stress, yield stress and flexural modulus) for each specimen. ${ }^{18,19}$

\section{Statistical analysis}

Data were presented as mean \pm standard deviation. The statistical differences among groups were evaluated using SPSS 16.0 software (SPSS Inc., Chicago, IL, USA) by analysis of 
variance with Fisher's protected least significant difference. A value of $P<0.05$ was considered statistically significant.

\section{Results}

$\mathrm{X}$-ray imaging revealed that healthy CD-1 mice (15-weekold) require $\sim 5$ weeks to complete the entire fracture healing process, whereas the mice with prednisone exposure needed $>9$ weeks (GC-M and GC-H) to complete the fracture healing process (Figure 1). Callus size analysis using the X-ray images further quantified the delayed fracture healing process in animals exposed to prednisone (GC-H, Figure 2).

Histological analysis of the fracture callus at 2 weeks post-closed femur fracture revealed that at the time of the necropsy, bone area and bone ratio in the TCA of mice exposed to prednisone clearly decreased, and the ratio of chondrocyte in the TCA and chondrocyte area were remarkably higher in mice exposed to prednisone than in those without prednisone exposure (Figure 3 and Table 1). Micro-CT analysis of the callus tissue further validated the histological finding. The quantitative analysis of the micro-CT data demonstrates that the callus BV/TV value of the mice with prednisone exposure was significantly lower than that of the mice without prednisone exposure. GC also significantly decreased BS/TV and BMC (Figure 4 and Table 2).

Analysis of the callus by micro-CT at 9 weeks postfracture showed that BV/TV of the callus tissue and BMD in mice with prednisone exposure were significantly lower than those in mice without prednisone exposure. Although callus size in the control group was smaller than that in the GC-H group, the callus in the control mice remodeled to normal cortical bone, while numerous woven bones still exist in the callus of mice exposed to GC (Figure 5).

Evaluation of the mechanical properties of the femur samples revealed that the structural strength (stiffness) and

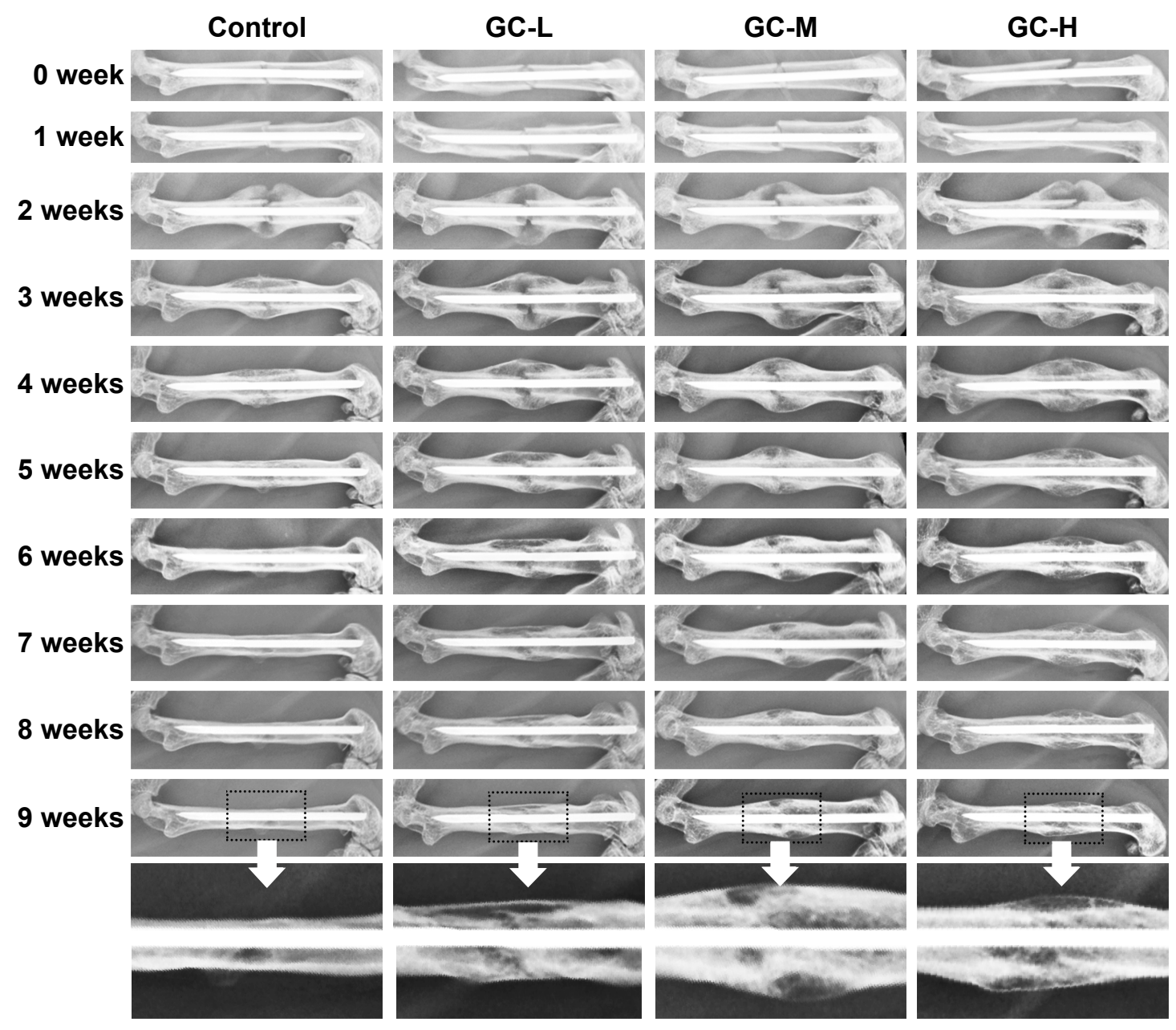

Figure I X-ray imaging of the fracture healing process in mice with prednisone exposure.

Notes: GC-L: CD-I mice exposed to prednisone (6 mg/kg/day); GC-M: CD-I mice exposed to prednisone ( $9 \mathrm{mg} / \mathrm{kg} / \mathrm{day})$; GC-H: CD-I mice exposed to prednisone ( $12 \mathrm{mg} / \mathrm{kg} /$ day). Control: wild-type CD-I mice. 

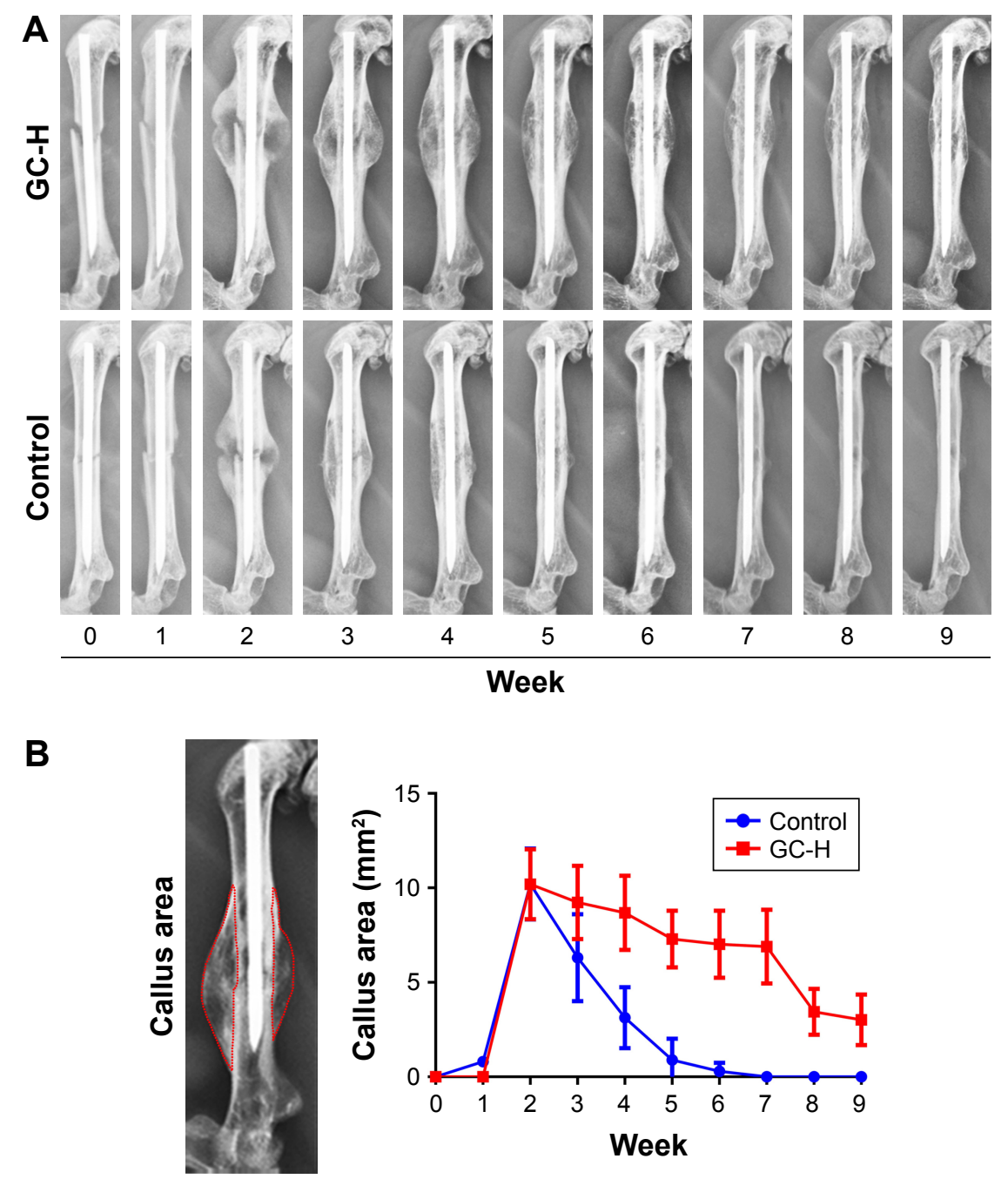

Figure $2 \mathrm{X}$-ray imaging analysis of fracture callus tissue post-fracture.

Notes: GC-H: CD-I mice exposed to prednisone ( $12 \mathrm{mg} / \mathrm{kg} /$ day); Control: wild-type CD-I mice. (A) Representative radiographs of the fracture healing process of mice in the GC-H and control groups. (B) Radiographs of fractured callus area in mice quantified using Imagej software.

apparent material strength (ultimate stress, yield stress and flexural modulus) decreased remarkably in mice with prednisone exposure compared with those in the healthy control (Table 3).

\section{Discussion}

The development of an appropriate delayed fracture healing animal model to investigate fracture healing pathophysiology requires proper selection of small animals that could rapidly heal their fractures. Mice are the first choice because of their short healing process (ie, $\sim 28$ days), with remodeling that continues for quite some time thereafter. In this study, X-ray images showed that the normal healing process in healthy CD-1 mice required $\sim 5$ weeks, whereas mice exposed to GC required $>9$ weeks. At the ninth week, mice exposed to GC radiographically appeared to be on the remodeling phase of healing, thereby indicating that the fracture had became an established delayed union. The use of the GC clearly impaired the process of normal bone healing. Appropriate and controlled chondrogenesis and endochondral ossification play fundamental roles in the fracture healing process. During the normal fracture healing, early intramembranous ossification is followed by endochondral ossification. In this study, endochondral ossification of chondrocytes in mice exposed to GC occurred much later than that in the normal control; hence, more chondrocytes were observed in the callus section of histological analysis from the mice exposed to prednisone. Micro-CT analysis also showed less mineral callus formation with decreased BMC and Conn.D than the control at 2 weeks post-fracture. Three point bending test at 9 weeks post-fracture revealed that there are significant differences between the fracture femurs from healthy control and 

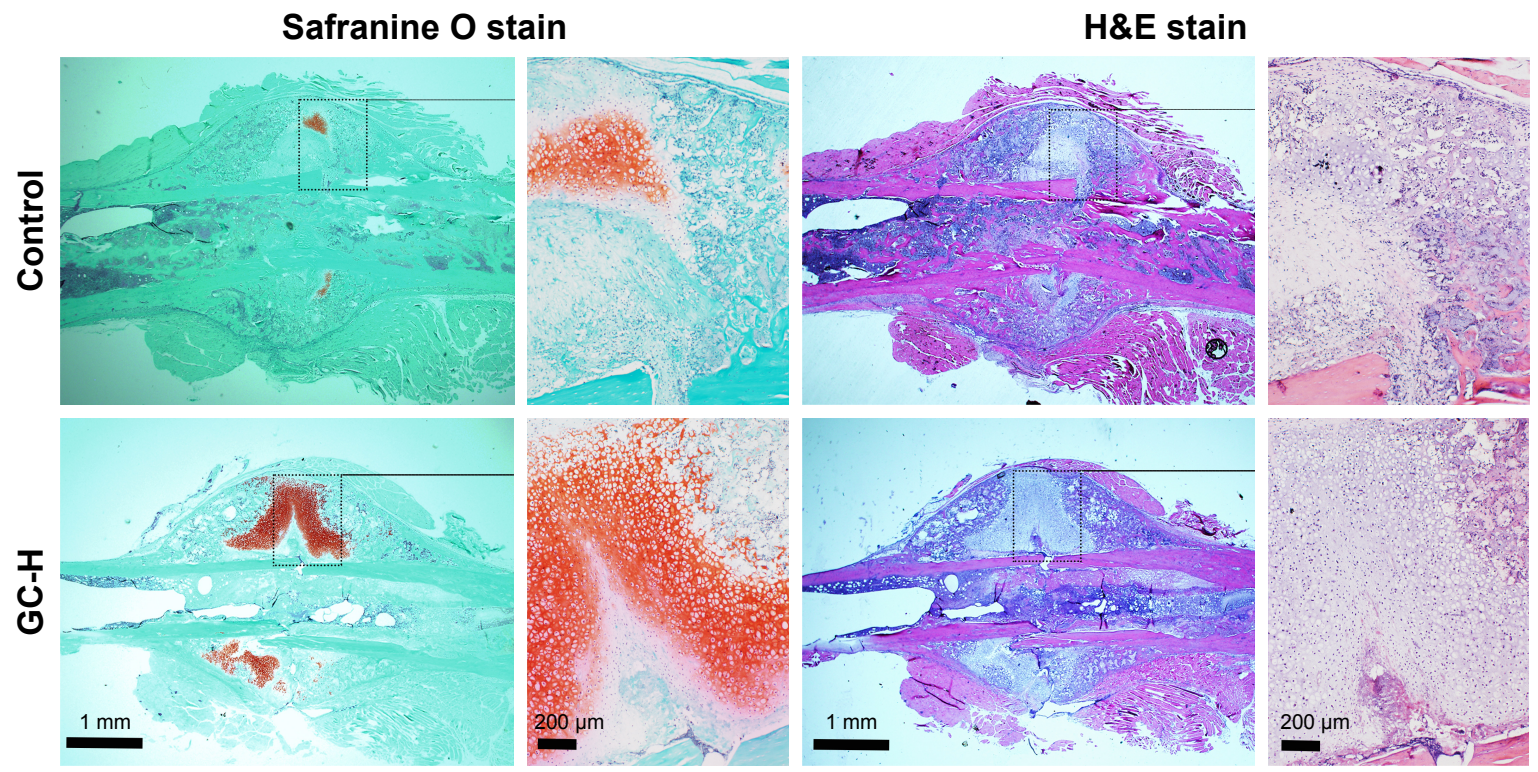

Figure 3 Representative histological images of fracture callus at 2 weeks post-closed femur fracture in mice.

Notes: GC-H: CD-I mice exposed to prednisone ( $12 \mathrm{mg} / \mathrm{kg} / \mathrm{day})$; Control: wild-type CD-I mice. Longitudinal sections of the calluses were subjected to Safranin O and H\&E staining. Red color indicates the chondrocyte area in the callus. Chondrocyte area, total callus area and bone area in the callus were quantified with Image-Pro Plus 6.0 based on histological staining (Table I).

Abbreviation: H\&E, hematoxylin and eosin.

the delayed fracture healing mice in most of the mechanical parameters tested. Micro-CT analysis also demonstrated that the fracture mice with GC exposure still had the remodeling woven callus around the fracture site, while the callus in the healthy control mice had already remodeled to cortical bone at 9 weeks post-fracture, which may explain the different mechanical properties between the healthy control and the mice with delayed fracture healing.

Although the molecular and cellular changes in the GCinduced delayed fracture healing process were not further investigated in this research, studies have demonstrated the negative effects of GCs on bone metabolism. For instance, GC-induced osteoporosis is fundamentally characterized by decreased bone remodeling, ${ }^{20}$ and the most predominant feature of the disease is decreased bone formation. Excessive

Table I Fracture callus quantified by histologic analysis at 2 weeks post-closed femur fracture

\begin{tabular}{lll}
\hline Variables & Control & GC-H \\
\hline Chondrocyte area $\left(\mathrm{mm}^{2}\right)$ & $0.29 \pm 0.23$ & $\mathrm{I} .65 \pm 0.87^{*}$ \\
Total callus area $\left(\mathrm{mm}^{2}\right)$ & $8.60 \pm 2.26$ & $8.01 \pm 2.44$ \\
Bone area $\left(\mathrm{mm}^{2}\right)$ & $3.49 \pm 0.73$ & $1.49 \pm 0.42^{*}$ \\
Bone/total callus (\%) & $40.86 \pm 7.48$ & $18.93 \pm 3.26^{*}$ \\
Chondrocyte/total callus (\%) & $3.47 \pm 2.0 \mathrm{I}$ & $20.08 \pm 4.67^{*}$ \\
\hline
\end{tabular}

Notes: $* P<0.05, G C-H$ : CD-I fracture mice exposed to prednisone $12 \mathrm{mg} / \mathrm{kg} / \mathrm{d}$; Control: healthy CD-I fracture mice. Chondrocyte area, total callus area and bone area in the callus were quantified with Image Pro Plus 6.0 based on histologic staining.
GCs directly decrease osteoblast number, induce apoptosis of mature osteoblasts ${ }^{21,22}$ and impair bone marrow stroma stem cell differentiation into mature functioning osteoblasts, thereby decreasing the number of bone-forming cells. GCs also suppress osteoblast function and activity, ${ }^{23,24}$ which in turn inhibits the synthesis of collagen and thus impairs osteoblastogenesis. In addition to the direct actions of GCs on osteoblasts, GCs also increase osteocyte apoptosis, decrease vascular endothelial growth factor, suppress skeletal angiogenesis and increase adipogenesis in the bone marrow, ${ }^{25}$ which further contribute to the loss of bone strength. ${ }^{26}$

GCs exert early direct effects (eg, elevating expression of RANKL and M-CSF) ${ }^{24,27,28}$ on osteoclasts to increase both their number and activity, with a corresponding increase in bone resorption. However, the long-term effects of GCs on osteoclasts remain controversial. ${ }^{29}$ Some studies revealed that a 27-day prednisolone treatment in mice result in decreased bone resorption below normal level. ${ }^{30}$ GCs were found to directly block the induction of cytoskeletal changes in osteoclasts, which are required for the resorptive capabilities of the cells. ${ }^{31}$ They also suppress the proliferation of osteoclast precursors. $^{32}$ These data indicate that GCs suppress both osteoblast and osteoclast function and thus decrease bone remodeling. The initial rapid loss of bone may reflect persistence of the prior effects of inflammatory cytokines, such as TNF- $\alpha$ and IL-1, as well as the osteoclastic cytokine RANKL. A previous study hypothesized that early in the course of 

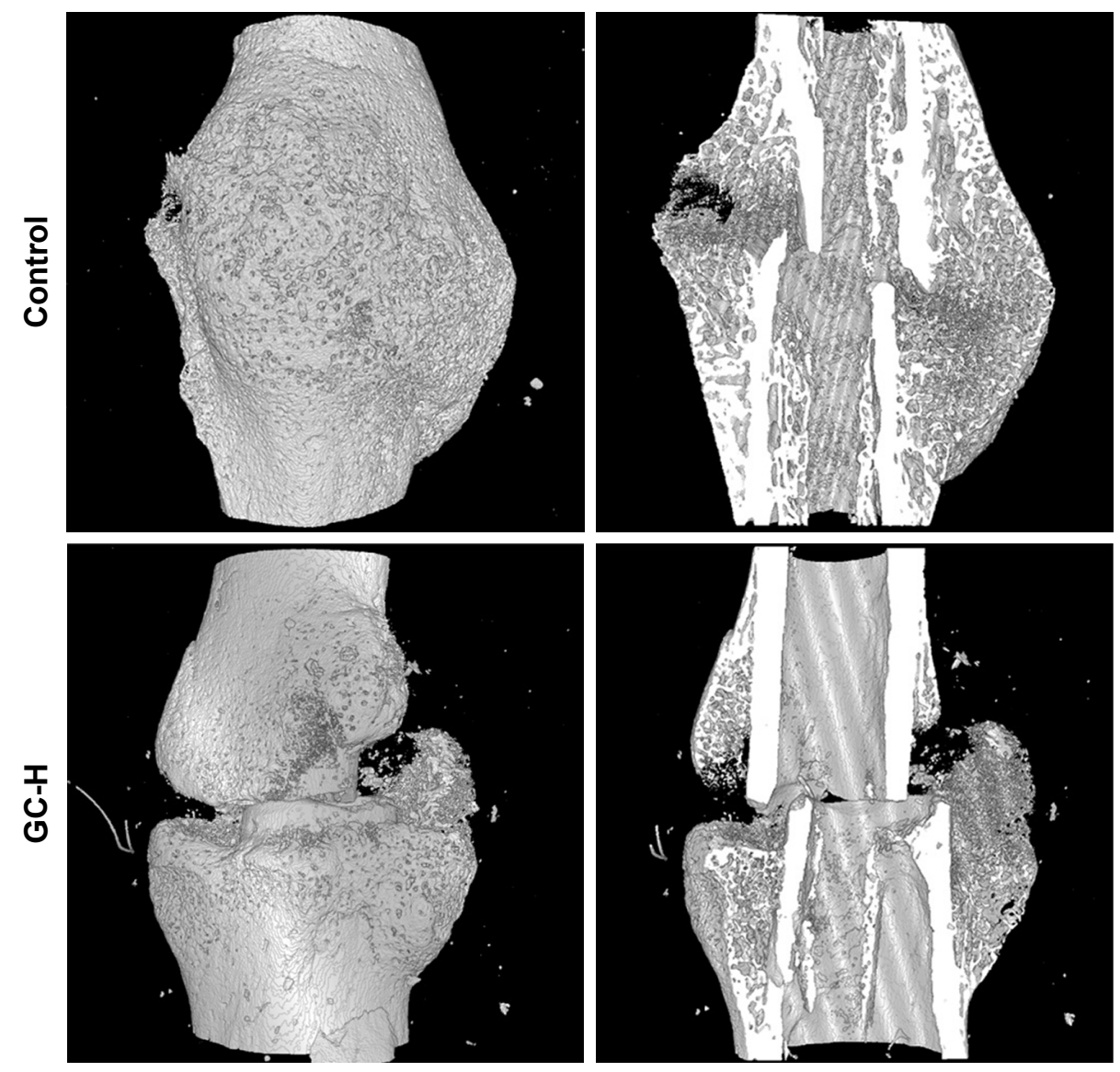

Figure 4 Micro-CT images of fractured femoral callus from representative specimens at 2 weeks post-fracture.

Notes: GC-H: CD-I fracture mice exposed to prednisone $12 \mathrm{mg} / \mathrm{kg} /$ day. Control: healthy CD-I fracture mice. Fracture callus at 2 weeks post-closed femur fracture was quantified by micro-CT (Table 2).

GC therapy, prior and persistent effects of inflammatory cytokines and RANKL override GC's inhibition on osteoclast function. After the early stage of persistent effects, the effects of GC inhibition on osteoclast function would become predominant. Moreover, bone resorption and formation are coupled. A significant suppression of osteoblastogenesis by GC may also impair osteoclastogenesis as the source of signals determining osteoclastogenesis is from osteoblasts. When the osteoblasts are significantly suppressed,

Table 2 Fracture callus quantified by micro-CT analysis at 2 weeks post-closed femur fracture

\begin{tabular}{lll}
\hline Parameter & Control & GC-H \\
\hline BV/TV $(\%)$ & $4.9 \pm 1.1$ & $3.7 \pm 1.3^{*}$ \\
BS/TV $(\mathrm{I} / \mathrm{mm})$ & $5.0 \pm 1.2$ & $3.7 \pm 1.2^{*}$ \\
Conn.D $\left(\mathrm{I} / \mathrm{mm}^{3}\right)$ & $442.1 \pm 86.8$ & $381.4 \pm 132.9$ \\
BMC $(\mathrm{mg})$ & $6.0 \pm 1.6$ & $3.6 \pm 1.4^{*}$ \\
\hline
\end{tabular}

Notes: $* P<0.05, \mathrm{GC}-\mathrm{H}$ : CD-I fracture mice exposed to prednisone $12 \mathrm{mg} / \mathrm{kg} / \mathrm{day}$; Control: healthy CD-I fracture mice.

Abbreviations: BV/TV, bone volume to tissue volume; $\mathrm{BS} / \mathrm{TV}$, callus bone surface to volume; Conn.D, connectivity density; BMC, bone mineral content. osteoblastic signals controlling osteoclastogenesis are also reduced, thereby decreasing the osteoclast number. ${ }^{33}$ In this study, since prednisone could inhibit the inflammatory responses, which is important for the osteoclastogenesis and osteoclast maturation, prednisone appears to suppress osteoclast number and function during the endochondral ossification stage and the bone remodeling stage. Therefore, the delayed fracture healing in GC-treated mice may partially attribute to the inhibition of osteoclastogenesis.

Although researches have reported different effects of GC on bone metabolism, the role of GC in the fracture healing process still requires extensive investigation. Genetic susceptibility factors, altered immune response, complicated cascade of bone regeneration and a series of different cell types are involved in the healing process, ${ }^{34}$ which provide GCs with more complicate targets than those in the osteoporosis. Further investigation on the specific role of GCs in the fracture healing would be interesting and challenging.

There may also be several limitations in this study. First, while the closed fracture strategy used in this model may 

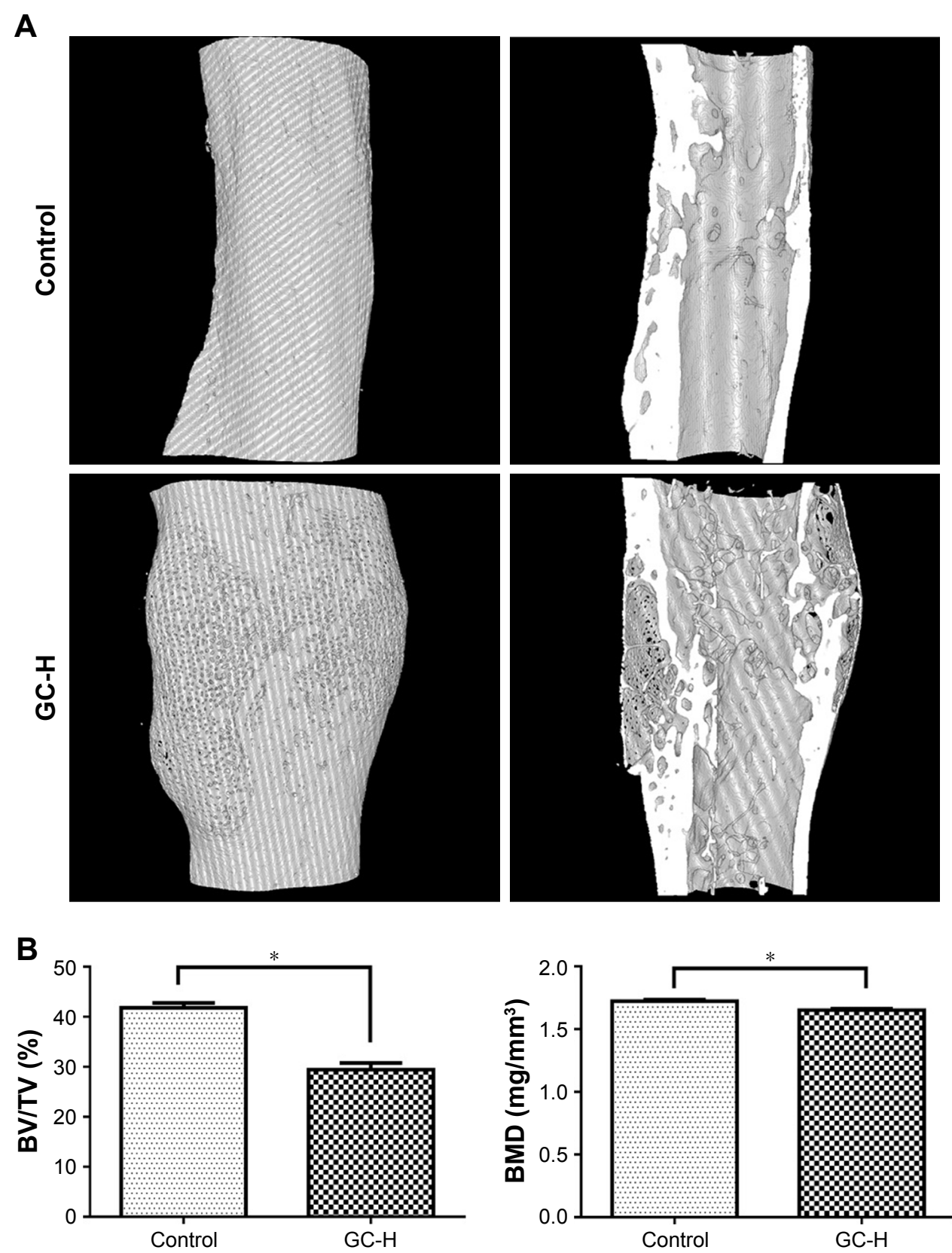

Figure 5 Micro-CT analysis of fractured femoral callus from representative specimens at 9 weeks post-fracture.

Notes: (A) Representative micro-CT images of fracture callus at 9 weeks post-closed femur fracture; (B) BMD and callus BV/TV were quantified by micro-CT analysis. $* P<0.05$; GC-H: CD-I fracture mice exposed to prednisone $12 \mathrm{mg} / \mathrm{kg} /$ day; Control: healthy $\mathrm{CD}$-I fracture mice.

Abbreviations: BMD, bone mineral density; BV/TV, bone volume to tissue volume.

Table 3 Analysis of the biomechanical properties of the healed femur at 9 weeks post-femur fracture

\begin{tabular}{lll}
\hline Variables & Control & GC-H \\
\hline Stiffness $(\mathrm{N} / \mathrm{mm})$ & $198.2 \pm 45.3$ & $130.2 \pm 33.5^{*}$ \\
Ultimate stress $\left(\mathrm{N} / \mathrm{mm}^{2}\right)$ & $195.6 \pm 33.1$ & $93.1 \pm 32.2^{*}$ \\
Yield stress $\left(\mathrm{N} / \mathrm{mm}^{2}\right)$ & $160.9 \pm 37.7$ & $82.1 \pm 30.5^{*}$ \\
Flexural modulus $\left(\mathrm{N} / \mathrm{mm}^{2}\right)$ & $1,823.2 \pm 548.5$ & $694.3 \pm 297 . \mathrm{I}^{*}$ \\
Toughness $(\mathrm{N}-\mathrm{mm})$ & $3.27 \pm 1.87$ & $2.48 \pm 1.34$ \\
Resiliance $(\mathrm{N}-\mathrm{mm})$ & $1.76 \pm 0.82$ & $1.51 \pm 0.92$ \\
\hline
\end{tabular}

Notes: $* P<0.05$, GC-H: CD-I fracture mice exposed to prednisone $12 \mathrm{mg} / \mathrm{kg} / \mathrm{d}$. Control: healthy CD-I fracture mice. offer some advantages (ie, simple surgical approach with limited skin and muscle damage), it cannot completely avoid axial and rotational instability. A few pin slippage to the knee may occur in mice after the fracture procedure. In GC-treated mice, as osteogenesis was inhibited by GCs, bone formation around the pin was decreased or delayed and the integration between pin and bone was weaker that control. A second surgery may need to cut off the slipped spin outside the cavity when pin slippage occurred. Locking nail or intramedullary 
compression screw may provide better fracture fixation. In that case, however, the fracture type would become an open fracture as the installation of locking nail or intramedullary compression screw would necessitate exposure of the fracture site with skin and muscle trauma. Second, we did not detect estrogen level in the female mice and match their estrogen cycle before the fracture procedure in this study. It is well recognized that estrogen affects skeletal quality and the fracture healing process. When compared to the chronic GC exposure, however, the impacts of the fluctuating estrogen level on the skeleton may not be as significant. Using male mice for this study may avoid the estrogen issue. Young female mice were used instead of male mice in this study because they could provide several advantages. 1) Practically, female mice are less aggressive than male mice. The more tampered behavior may help to reduce infighting among the mice and avoid delayed healing caused by instability at the fracture site. 2) For some systemic autoimmune diseases, such as lupus and rheumatoid arthritis, there is a higher incidence rate among young female than male or other age of population. These conditions are treated with long-term GCs. Therefore, the selection of young female mice for establishing the delayed fracture healing model is clinically relevant. 3) Young mice have normal osteogenesis ability. When GC is used to create the delayed healing model, the delayed healing may be solely attributed to the GC exposure, rather than aging or estrogen deficiency. 4) Young mice also have a shorter cycle for fracture healing, which may help to reduce the time needed for each experiment.

In conclusion, here we have reported a reproducible delayed femur fracture healing mouse model that may partially recapitulate a clinical pathology of impaired bone fracture healing due to GC exposure. The model features delayed union according to radiographic evaluation, reduced endochondral ossification and significantly decreased biomechanical properties. Although this delayed fracture healing model may not fully capture the effect of GCs on fracture healing process, it demonstrates close similarities with several significant histopathological aspects of fracture healing impairment in humans. Therefore, this model may be used not only to systematically study the cellular and molecular mechanisms underlying fracture healing with GCs exposure, but also for drug screening and development of therapeutic interventions, which may augment the bone fracture healing process.

\section{Acknowledgments}

This study was supported in part by the National Natural Science Foundation of China (grant no 81703584),
Guangdong Province Natural Science Foundation of China (grant no 2017A030310614), Science and Technology Competitive Financial Fund of Zhanjiang (grants no 2016A01012 and 2017A01021), the National Institute of Arthritis and Musculoskeletal and Skin Diseases of the National Institute of Health of the United States of America (R01AR062680), the University of Nebraska Medical Center College of Pharmacy and Guangdong Medical University scientific research fund (grant no B2017001).

\section{Author contributions}

Liao Cui, Dong Wang and Yan-zhi Liu designed this study; Yan-zhi Liu, Xiao-yan Wang and Xiao-bei Wang carried out the experiments; Mohammed P Akhter performed the biomechanical property analysis; Xiao Gao performed the micro-CT analysis; Gang Zhao and Xin Wei contributed to histological analysis, Hao-jun Wu and Hang Chen analyzed the X-ray images; Yan-zhi Liu, Liao Cui, and Dong Wang prepared the manuscript. All the authors read and approved the final manuscript.

\section{Disclosure}

The authors report no conflicts of interest in this work.

\section{References}

1. Yelin E, Weinstein S, King T. The burden of musculoskeletal diseases in the United States. Semin Arthritis Rheum. 2016;46(3):259-260.

2. Antonova E, Le TK, Burge R, Mershon J. Tibia shaft fractures: costly burden of nonunions. BMC Musculoskelet Disord. 2013;14:42.

3. Gaston MS, Simpson AH. Inhibition of fracture healing. J Bone Joint Surg Br. 2007;89(12):1553-1560.

4. Overman RA, Yeh JY, Deal CL. Prevalence of oral glucocorticoid usage in the United States: a general population perspective. Arthritis Care Res (Hoboken). 2013;65(2):294-298.

5. Fardet L, Petersen I, Nazareth I. Prevalence of long-term oral glucocorticoid prescriptions in the UK over the past 20 years. Rheumatology (Oxford). 2011;50(11):1982-1990.

6. van Staa TP, Leufkens HG, Abenhaim L, Begaud B, Zhang B, Cooper C. Use of oral corticosteroids in the United Kingdom. QJM. 2000;93(2):105-111.

7. Fardet L, Petersen I, Nazareth I. [Description of oral glucocorticoid prescriptions in general population]. Rev Med Interne. 2011;32(10): 594-599. French [with English abstract].

8. Waters RV, Gamradt SC, Asnis P, et al. Systemic corticosteroids inhibit bone healing in a rabbit ulnar osteotomy model. Acta Orthop Scand. 2000;71(3):316-321.

9. Sawin PD, Dickman CA, Crawford NR, Melton MS, Bichard WD, Sonntag VK. The effects of dexamethasone on bone fusion in an experimental model of posterolateral lumbar spinal arthrodesis. J Neurosurg. 2001;94(1 Suppl):76-81.

10. Ehrlich HP, Tarver H, Hunt TK. Effects of vitamin A and glucocorticoids upon inflammation and collagen synthesis. Ann Surg. 1973;177(2): 222-227.

11. Duthie RB, Barker AN. The histochemistry of the preosseous stage of bone repair studied by autoradiography; the effect of cortisone. $J$ Bone Joint Surg Br. 1955;37-B(4):691-700. 
12. Aslan M, Simşek G, Yildirim U. Effects of short-term treatment with systemic prednisone on bone healing: an experimental study in rats. Dent Traumatol. 2005;21(4):222-225.

13. Høgevold HE, Grøgaard B, Reikerås O. Effects of short-term treatment with corticosteroids and indomethacin on bone healing. A mechanical study of osteotomies in rats. Acta Orthop Scand. 1992;63(6): $607-611$.

14. Key JA, Odell RT, Taylor LW. Failure of cortisone to delay or to prevent the healing of fractures in rats. J Bone Joint Surg Am. 1952; 24 A(3):665-675; passim.

15. Pountos I, Georgouli T, Blokhuis TJ, Pape HC, Giannoudis PV. Pharmacological agents and impairment of fracture healing: what is the evidence? Injury. 2008;39(4):384-394.

16. Zhang Y, Jia Z, Yuan H, et al. The evaluation of therapeutic efficacy and safety profile of simvastatin prodrug micelles in a closed fracture mouse model. Pharm Res. 2016;33(8):1959-1971.

17. Bonnarens F, Einhorn TA. Production of a standard closed fracture in laboratory animal bone. J Orthop Res. 1984;2(1):97-101.

18. Akhter MP, Wells DJ, Short SJ, et al. Bone biomechanical properties in LRP5 mutant mice. Bone. 2004;35(1):162-169.

19. Akhter MP, Cullen DM, Pan LC. Bone biomechanical properties in EP4 knockout mice. Calci Tissue Int. 2006;78(6):357-362.

20. Canalis E, Delany AM. Mechanisms of glucocorticoid action in bone. Ann N Y Acad Sci. 2002;966:73-81.

21. Canalis E, Mazziotti G, Giustina A, Bilezikian JP. Glucocorticoidinduced osteoporosis: pathophysiology and therapy. Osteoporos Int. 2007;18(10):1319-1328.

22. Weinstein RS. Clinical practice. Glucocorticoid-induced bone disease. $N$ Engl J Med. 2011;365(1):62-70.

23. Liu Y, Cui Y, Chen Y, Gao X, Su Y, Cui L. Effects of dexamethasone, celecoxib, and methotrexate on the histology and metabolism of bone tissue in healthy Sprague Dawley rats. Clin Interv Aging. 2015;10:1245-1253.
24. Liu Y, Cui Y, Zhang X, et al. Effects of salvianolate on bone metabolism in glucocorticoid-treated lupus-prone B6.MRL-Fas (lpr)/J mice. Drug Des Devel Ther. 2016;10:2535-2546.

25. Cui L, Li T, Liu Y, et al. Salvianolic acid B prevents bone loss in prednisone-treated rats through stimulation of osteogenesis and bone marrow angiogenesis. PLoS One. 2012;7(4):e34647.

26. Weinstein RS, Wan C, Liu Q, et al. Endogenous glucocorticoids decrease skeletal angiogenesis, vascularity, hydration, and strength in aged mice. Aging Cell. 2010;9(2):147-161.

27. Dovio A, Perazzolo L, Saba L, et al. High-dose glucocorticoids increase serum levels of soluble IL-6 receptor alpha and its ratio to soluble gp130: an additional mechanism for early increased bone resorption. Eur J Endocrinol. 2006;154(5):745-751.

28. Rubin J, Biskobing DM, Jadhav L, et al. Dexamethasone promotes expression of membrane-bound macrophage colony-stimulating factor in murine osteoblast-like cells. Endocrinology. 1998;139(3): 1006-1012.

29. Teitelbaum SL. Glucocorticoids and the osteoclast. Clin Exp Rheumatol. 2015;33(4 Suppl 92):S37-S39.

30. Weinstein RS, Jilka RL, Parfitt AM, Manolagas SC. Inhibition of osteoblastogenesis and promotion of apoptosis of osteoblasts and osteocytes by glucocorticoids. Potential mechanisms of their deleterious effects on bone. J Clin Invest. 1998;102(2):274-282.

31. Kim HJ, Zhao H, Kitaura H, et al. Glucocorticoids and the osteoclast. Ann N Y Acad Sci. 2007;1116:335-339.

32. Kim HJ, Zhao H, Kitaura H, et al. Glucocorticoids suppress bone formation via the osteoclast. J Clin Invest. 2006;116(8):2152-2160.

33. Jia D, O'Brien CA, Stewart SA, Manolagas SC, Weinstein RS. Glucocorticoids act directly on osteoclasts to increase their life span and reduce bone density. Endocrinology. 2006;147(12):5592-5599.

34. Hankenson KD, Zimmerman G, Marcucio R. Biological perspectives of delayed fracture healing. Injury. 2014;45 Supp1 2:S8-S15.
Clinical Interventions in Aging

\section{Publish your work in this journal}

Clinical Interventions in Aging is an international, peer-reviewed journal focusing on evidence-based reports on the value or lack thereof of treatments intended to prevent or delay the onset of maladaptive correlates of aging in human beings. This journal is indexed on PubMed Central, MedLine,

\section{Dovepress}

CAS, Scopus and the Elsevier Bibliographic databases. The manuscript management system is completely online and includes a very quick and fair peer-review system, which is all easy to use. Visit http://www.dovepress. com/testimonials.php to read real quotes from published authors. 